\title{
TANGENT CONES AND QUASI-INTERIORLY TANGENT CONES TO MULTIFUNCTIONS
}

BY

\author{
LIONEL THIBAULT
}

\begin{abstract}
R. T. Rockafellar has proved a number of rules of subdifferential calculus for nonlocally lipschitzian real-valued functions by investigating the Clarke tangent cones to the epigraphs of such functions. Following these lines we study in this paper the tangent cones to the sum and the composition of two multifunctions. This will be made possible thanks to the notion of quasi-interiorly tangent cone which has been introduced by the author for vector-valued functions in [29] and whose properties in the context of multifunctions are studied. The results are strong enough to cover the cases of real-valued or vector-valued functions.
\end{abstract}

Introduction. Rockafellar has introduced in [23] the very important notion of directionally lipschitzian behaviour for extended real-valued functions, and with the aid of this notion he has proved in [24] a number of rules of subgradient calculus of nonconvex functions. If $f$ is a function from a topological vector space $E$ into $\mathbf{R} \cup\{-\infty,+\infty\}$ with $f(\bar{x}) \in \mathbf{R}$ and if $I($ epi $f ; \bar{x}, f(\bar{x}))$ denotes the interiorly tangent cone to

$$
\text { epi } f=\{(x, y) \in E \times \mathbf{R}: f(x) \leqslant y\}
$$

at $(\bar{x}, f(\bar{x}))$, that is, the set of all $(v, w) \in E \times \mathbf{R}$ such that there exist a neighbourhood $X$ of $(\bar{x}, f(\bar{x}))$, a neighbourhood $V$ of $(v, w)$ in $E \times \mathbf{R}$ and a real number $\varepsilon>0$ such that $X \cap$ epi $f+] 0, \varepsilon[V \subset$ epi $f$, then the proof of Theorem 3 of [23, p. 268] shows that $f$ is directionally lipschitzian at $\bar{x}$ if and only if $I($ epi $f ; \bar{x}, f(\bar{x})) \neq \varnothing$. However, if $g$ is a mapping from $E$ into an ordered topological vector space $H$, then the interior of the cone of positive elements of $H$ must be nonempty whenever $I($ epi $g ; \bar{x}, g(\bar{x}))$ is nonempty. This very unsatisfactory state of affairs has led us to introduce in [29 and 30] the quasi-interiorly tangent cone $Q($ epi $g ; \bar{x}, g(\bar{x}))$. With the help of this cone we have established in [29] rules of subdifferential calculus for nonconvex vector-valued functions. The aim of the present paper is to study the properties of Clarke tangent cones and quasi-interiorly tangent cones to the graphs of multifunctions following the way opened by Rockafellar.

In $\$ 1$ we recall Rockafellar's definition of Clarke tangent cone and we give an interpretation in terms of generalized sequences which proves that Rockafellar's definition is the same as the one we have given in [27]. Connection with strictly compactly lipschitzian vector-valued mappings is also made.

Received by the editors March 19, 1982 and, in revised form, May 10, 1982.

1980 Mathematics Subject Classification. Primary 90C30, 90C48; Secondary 58C06.

Key words and phrases. Tangent cones, quasi-interiorly tangent cones, convex and lipschitzian multifunctions, additively separate multifunctions. 
$\S 2$ is devoted to the study of quasi-interiorly tangent cones to graphs of multifunctions and to the relationships between these cones and Clarke tangent cones. We prove that the quasi-interiorly tangent cone is always convex and that the Clarke tangent cone is the closure of the quasi-interiorly tangent cone whenever the quasi-interiorly tangent cone is nonempty. The cases of lipschitzian multifunctions and convex multifunctions are also considered.

The final two sections deal with the study of the sum of two multifunctions and of the composition of a multifunction with a differentiable mapping. The results are strong enough to cover the corresponding ones given in [ 24 and 29] for real-valued and vector-valued functions.

Although the details will not be given here, this way to approach multifunctions by convex multifunctions can be used to study the existence of Lagrange multipliers for programming problems with constraints defined by multifunctions (see [31]). To conclude this introduction let us indicate that Bouligand tangent cones to multifunctions have been considered in [ 1 and 15] (see also [7 and 14]).

1. Tangent cones. In this paper all topological spaces will be assumed to be Hausdorff and $E$ and $F$ will denote two topological vector spaces.

Let $M$ be a multifunction from a topological space $S$ into another one $T$, that is, $M(s)$ is a subset of $E$ (possibly empty) for each $s \in S$. We shall consider in the sequel the graph of $M$,

$$
\operatorname{Gr} M=\{(s, t) \in S \times T: t \in M(s)\},
$$

and the domain of $M$,

$$
\operatorname{dom} M=\{s \in S: M(s) \neq \varnothing\} .
$$

If $D$ is a nonempty subset of $S$ and if $\bar{s}$ is a point in the closure of $D$ in $S$, we recall that the lower limit of $M$ as $s \in D \rightarrow \bar{s}$ is the set $\liminf _{s \rightarrow^{D_{s}}} M(s)$ of all $t \in T$ such that for each neighbourhood $W$ of $t$ in $T$ there exists a neighbourhood $V$ of $\bar{s}$ in $S$ such that

$$
W \cap M(s) \neq \varnothing
$$

for all $s \in D \cap V$ (see [2 and 23]).

As sequential motivation for tangent cones, we shall give a characterization of the above limit in terms of nets (generalized sequences).

Let $\left(s_{j}\right)_{j \in J}$ be a net (or generalized sequence) in $S$, that is, a family of points of $S$ indexed by a set $J$ which is directed by a preorder relation $\leqslant$ (that is to say that for each $\left(j_{1}, j_{2}\right) \in J \times J$ there exists $j_{3} \in J$ such that $j_{1} \leqslant j_{3}$ and $\left.j_{2} \leqslant j_{3}\right)$. By a subnet of $\left(s_{j}\right)_{j \in J}$ we shall mean (see [13]) a net $\left(s_{\alpha(i)}\right)_{i \in I}$ where $\alpha$ is a mapping from a directed set $I$ into $J$ such that for each $j \in J$ there is $i_{j} \in I$ such that $j \leqslant \alpha(i)$ for all $i \in I$ satisfying $i_{j} \leqslant i$.

1.1 Proposition. The lower limit $\liminf _{s^{D_{\bar{s}}}} M(s)$ is the set of all $t \in T$ such that for each net $\left(s_{j}\right)_{j \in J}$ of $D$ converging to $\bar{s}$ there exist a subnet $\left(s_{\alpha(i)}\right)_{i \in I}$ and a net $\left(t_{i}\right)_{i \in I}$ converging to $t$ such that $t_{i} \in M\left(s_{\alpha(i)}\right)$ for all $i \in I$. 
Proof. Consider $\bar{t} \notin \liminf _{s \rightarrow{ }^{D} \bar{s}} M(s)$. There exists a neighbourhood $W$ of $\bar{t}$ in $T$ such that for each neighbourhood $V$ of $\bar{s}$ in $S$ there is a point $s_{V} \in V \cap D$ verifying

$$
W \cap M\left(s_{V}\right)=\varnothing \text {. }
$$

If the set $J$ of all neighbourhoods of $\bar{s}$ is directed by the inclusion preorder $\left(V_{1} \geqslant V_{2}\right.$ if $\left.V_{1} \subset V_{2}\right)$, then (2) implies that there is no net $\left(t_{i}\right)_{i \in I}$ satisfying the assumption of the proposition.

Now suppose that $\bar{t} \in \liminf _{s \rightarrow{ }^{D}} M(s)$. Let $\left(s_{j}\right)_{j \in J}$ be a net of $D$ converging to $\bar{s}$ and let $W$ be a neighbourhood of $\bar{t}$ in $T$. There exists a neighbourhood $V_{W}$ of $\bar{s}$ in $S$ such that

$$
W \cap M(s) \neq \varnothing \quad \text { for all } s \in D \cap V_{W} .
$$

Since $\lim _{j \in J} s_{j}=\bar{s}$, there is $j_{W} \in J$ such that $s_{j} \in D \cap V_{W}$ for all $j \geqslant j_{W}$. Therefore for $j \geqslant j_{W}$ we may choose by (3) a point $t_{j, W} \in W$ satisfying $t_{j, W} \in M\left(s_{j}\right)$. Put

$$
I=\left\{(j, W): W \in \Re(\bar{t}), j \in J, j \geqslant j_{W}\right\},
$$

where $\mathscr{T}(\bar{t})$ denotes the set of all neighbourhoods of $\bar{t}$ in $T$, and direct $I$ by setting

$$
\left(j_{1}, W_{1}\right) \leqslant\left(j_{2}, W_{2}\right) \text { if } j_{1} \leqslant j_{2} \text { and } W_{2} \subset W_{1} \text {. }
$$

Consider the mapping $\alpha$ from $I$ into $J$ defined by $\alpha(i)=j$ if $i=(j, W)$. It is not difficult to see that $\left(s_{\alpha(i)}\right)_{i \in I}$ is a subnet of $\left(s_{j}\right)_{j \in J}$ such that

$$
\lim _{i \in I} t_{i}=\bar{t} \text { and } t_{i} \in M\left(s_{\alpha(i)}\right) \text { for all } i \in I \text {. }
$$

So the point $\bar{t}$ satisfies the assumption of the proposition and hence the proof is complete.

REMARK. If $S$ and $T$ are metrizable, the above proof also shows that a point $t$ is in $\liminf _{s \rightarrow{ }^{D}} M(s)$ if and only if for each sequence $\left(s_{n}\right)_{n \in \mathrm{N}}$ of $D$ converging to $\bar{s}$ there exists a sequence $\left(t_{n}\right)_{n \in \mathbf{N}}$ converging to $t$ such that $t_{n} \in M\left(s_{n}\right)$ for all $n \in \mathbf{N}$.

We shall study some properties of (Clarke) tangent cones to graphs of multifunctions. For this reason we shall begin by recalling Rockafellar's formulation of the (Clarke) tangent cone (see [23]).

1.2 Definition. Let $A$ be a subset of the topological vector space $E$. The tangent cone $T(A ; \bar{x})$ to $A$ at $\bar{x} \in \operatorname{cl}_{E} A$ is the set of all points $v \in E$ such that for every neighbourhood $V$ of $v$ in $E$ there exist a neighbourhood $X$ of $\bar{x}$ in $E$ and a real number $\varepsilon>0$ such that $(x+t V) \cap A \neq \varnothing$ for all $x \in X \cap A$ and $t \in] 0, \varepsilon[$.

$T(A ; \bar{x})$ is a closed convex cone in $E$ (see [23]) and

$$
T(A ; \bar{x})=\liminf _{\substack{x \rightarrow A \bar{x} \\ t \downarrow 0}} t^{-1}(A-x),
$$

where $x \rightarrow^{A} \bar{x}$ means $x \rightarrow \bar{x}$ and $x \in A$.

As an immediate consequence of Proposition 1.1 we have the following characterization of $T(A ; \bar{x})$ in terms of nets. This characterization is exactly the definition which has been given by the author in [27].

1.3 Proposition. $T(A ; \bar{x})$ is the set of all $v \in E$ such that for every net $\left(x_{j}\right)_{j \in J}$ in $A$ converging to $\bar{x}$ and every net $\left(t_{j}\right)_{j \in J}$ of positive numbers converging to zero there exist 
two subnets $\left(x_{\alpha(i)}\right)_{i \in I}$ and $\left(t_{\alpha(i)}\right)_{i \in I}$ and a net $\left(v_{i}\right)_{i \in I}$ in $E$ converging to $v$ such that $x_{\alpha(i)}+t_{\alpha(i)} v_{i} \in A$ for each $i \in I$.

REMARK. If $E$ and $F$ are metrizable topological vector spaces we may replace, in the above proposition, nets and subnets by sequences and subsequences, and we get the formulation given by the author in [25], or only by sequences and we get the formulation given by Hiriart-Urruty in [10].

If $M$ is a multifunction from $E$ into $F$ with $(\bar{x}, \bar{y}) \in \operatorname{Gr} M$, we shall use the notations

$$
T(M ; \bar{x}, \bar{y}):=T(\operatorname{Gr} M ;(\bar{x}, \bar{y}))
$$

and

$$
T(M ; \bar{x}, \bar{y})(v):=\{w \in F:(v, w) \in T(M ; \bar{x}, \bar{y})\}
$$

for each $v \in E$. Moreover, we shall conventionally put $M(x)+\varnothing=\varnothing$ for each $x \in E$.

1.4 Proposition. Let $M$ be a multifunction from the topological vector space $E$ into the topological vector space $F$ with $\bar{y} \in M(\bar{x})$. If we put

$$
L(v)=\left\{\lim _{j \in J} t_{j}^{-1}\left(z_{j}-\bar{y}\right): z_{j} \in M\left(\bar{x}+t_{j} v_{j}\right), v_{j} \rightarrow v \text { and } t_{j} \downarrow 0\right\},
$$

then $T(M ; \bar{x}, \bar{y})(v) \subset L(v)$.

Proof. Let $(v, w) \in T(M ; \bar{x}, \bar{y})$. If $\left(t_{j}\right)_{j \in J}$ is a net of positive numbers converging to zero, then by Proposition 1.1 there exist a subnet $\left(t_{\alpha(i)}\right)_{i \in I}$ and a net $\left(v_{i}, w_{i}\right)_{i \in I}$ converging to $(v, w)$ such that $(\bar{x}, \bar{y})+t_{\alpha(i)}\left(v_{i}, w_{i}\right) \in \operatorname{Gr} M$ and hence

$$
z_{i}:=\bar{y}+t_{\alpha(i)} w_{i} \in M\left(\bar{x}+t_{\alpha(i)} v_{i}\right)
$$

and

$$
w=\lim _{i \in I} w_{i}=\lim _{i \in I} t_{\alpha(i)}^{-1}\left(z_{i}-\bar{y}\right) .
$$

Let us recall the definition of strictly compactly lipschitzian mappings.

1.5 Definition (SEE [26]). A mapping $f$ from $E$ into $F$ is strictly compactly lipschitzian at $\bar{x} \in E$ if there exist a mapping $K$ from $E$ into the $\operatorname{set} \operatorname{comp}(F)$ of nonempty compact subsets of $F$, a mapping $r$ from $] 0,1] \times E \times E$ into $F$, and neighbourhoods $X$ of $\bar{x}$ and $V$ of zero in $E$ satisfying:

(a) $\lim _{t \downarrow 0 ; x \rightarrow \bar{x}} r(t, x ; v)=0$ for each $v \in E$ and $\lim _{t \downarrow 0 ;(x, v) \rightarrow(\bar{x}, 0)} r(t, x ; v)=0$;

(b) for all $x \in X, v \in V$ and $t \in] 0,1]$,

$$
t^{-1}[f(x+t v)-f(x)] \in K(v)+r(t, x ; v)
$$

(c) $K(0)=\{0\}$ and the multifunction $K$ is upper semicontinuous at zero (that is, for every neighbourhood $W$ of $K(0)$ in $F$ there is a neighbourhood $U$ of zero in $E$ satisfying $K(v) \subset W$ for every $v \in U)$.

REMARKS (SEE [26]). If $f$ is strictly compactly lipschitzian at $\bar{x}$, then the following properties are satisfied:

(1) $\lim _{t \downarrow 0 ;(x, w) \rightarrow(\bar{x}, v)} t^{-1}[f(x+t w)-f(x+t v)]=0$ for each $v \in E$; 
(2) for each $v \in E$, for each net $\left(x_{j}\right)_{j \in J}$ converging to $\bar{x}$ in $E$ and for each net $\left(t_{j}\right)_{j \in J}$ of positive numbers converging to zero, there exists a subnet

$$
\left(t_{\alpha(i)}^{-1}\left[f\left(x_{\alpha(i)}+t_{\alpha(i)} v\right)-f\left(x_{\alpha(i)}\right)\right]\right)_{i \in I}
$$

which converges;

(3) the mapping $f$ is continuous at $\bar{x}$.

1.6 Proposition. Let $P$ be a closed convex cone in $F$ and let $f$ be a mapping from $E$ into $F$ which is strictly compactly lipschitzian at $\bar{x} \in E$. For the multifunction $M$ from $E$ into $F$ defined by $M(x)=f(x)+P$ for every $x \in E$, and for $\bar{y}=f(\bar{x})$ we have

$$
T(M ; \bar{x}, \bar{y})(v)=\bigcap_{l \in L(v)}(l+P),
$$

where

$$
L(v)=\left\{\lim _{j \in J} q_{f}\left(t_{j}, x_{j} ; v\right): t_{j} \downarrow 0, x_{j} \rightarrow \bar{x}\right\}
$$

with $q_{f}(t, x ; v)=t^{-1}[f(x+t v)-f(x)]$.

Proof. Let $w$ be a point in $T(M ; \bar{x}, \bar{y})(v)$ and let $l=\lim _{j \in J} q_{f}\left(t_{j}, x_{j} ; v\right)$. As the net $\left(x_{j}, f\left(x_{j}\right)\right)_{j \in J}$ converges to $(\bar{x}, f(\bar{x}))$ (see Remark 3 following Definition 1.5) and as $\left(x_{j}, f\left(x_{j}\right)\right) \in \operatorname{Gr} M$, there exist two subnets, $\left(x_{\alpha(i)}, f\left(x_{\alpha(i)}\right)\right)_{i \in I}$ and $\left(t_{\alpha(i)}\right)_{i \in I}$, and a net $\left(v_{i}, w_{i}\right)_{i \in I}$ converging to $(v, w)$ such that

$$
\left(x_{\alpha(i)}, f\left(x_{\alpha(i)}\right)\right)+t_{\alpha(i)}\left(v_{i}, w_{i}\right) \in \mathrm{Gr} M
$$

for every $i \in I$. Therefore we have

$$
w_{i}-t_{\alpha(i)}^{-1}\left[f\left(x_{\alpha(i)}+t_{\alpha(i)} v_{i}\right)-f\left(x_{\alpha(i)}\right)\right] \in P,
$$

and, hence, by Remark 1 following Definition 1.5, w $\in l+P$. This proves that

$$
T(M ; \bar{x}, \bar{y})(v) \subset \bigcap_{l \in L(v)}(l+P) .
$$

Let us show the reverse inclusion. Let $w \in \bigcap_{l \in L(v)}(l+P)$. Consider a net $\left(x_{j}, y_{j}\right)_{j \in J}$ in $\operatorname{Gr} M$ converging to $(\bar{x}, \bar{y})$ and a net $\left(t_{j}\right)_{j \in J}$ of positive numbers converging to zero. Since $f$ is strictly compactly lipschitzian at $\bar{x}$, there exists a subnet

$$
\left(t_{\alpha(i)}^{-1}\left[f\left(x_{\alpha(i)}+t_{\alpha(i)} v\right)-f\left(x_{\alpha(i)}\right)\right]\right)_{i \in I}
$$

which converges (see Remark 2 following Definition 1.5). Put

$$
t_{\alpha(i)}^{-1}\left[f\left(x_{\alpha(i)}+t_{\alpha(i)} v\right)-f\left(x_{\alpha(i)}\right)\right]=\bar{l}+r_{i}
$$

with $\lim _{i \in I} r_{i}=0$. Then we have

$$
y_{\alpha(i)}+t_{\alpha(i)}\left(w+r_{i}\right) \in f\left(x_{\alpha(i)}\right)+P+t_{\alpha(i)}\left(\bar{l}+P+r_{i}\right)=f\left(x_{\alpha(i)}+t_{\alpha(i)} v\right)+P,
$$

and hence the net $\left(v, w+r_{i}\right)_{i \in I}$ converges to $(v, w)$ and satisfies

$$
\left(x_{\alpha(i)}, y_{\alpha(i)}\right)+t_{\alpha(i)}\left(v, w+r_{i}\right) \in \mathrm{Gr} M .
$$

Therefore $(v, w) \in T(M ;(\bar{x}, \bar{y}))$, and the proof of the proposition is finished. 
REMARK. If $f$ is strictly compactly lipschitzian at $\bar{x}$ in the direction $\bar{v} \in E$, that is, if there exist a compact $K(\bar{v})$ in $F$, a mapping $r(\cdot, \cdot ; \bar{v})$ from $] 0, \infty[\times E$ into $F$, a neighbourhood $X$ of $\bar{x}$ in $E$ and a number $\varepsilon>0$ satisfying:

(a) $\lim _{t \downarrow 0 ; x \rightarrow \bar{x}} r(t, x ; \bar{v})=0$;

(b) for all $x \in X, t \in] 0, \varepsilon], t^{-1}[f(x+t \bar{v})-f(x)] \in K(\bar{v})+r(t, x ; \bar{v})$;

(c) $\lim _{t \downarrow 0 ;(x, v) \rightarrow(\bar{x}, \bar{v})} t^{-1}[f(x+t v)-f(x+t \bar{v})]=0$;

then the above proof also shows that

$$
T(M ; \bar{x}, \bar{y})(\bar{v})=\bigcap_{l \in L(\bar{v})}(l+P) .
$$

As an immediate consequence of the above proposition we have the following result.

1.7 COROLlaRY. If a mapping $f$ from $E$ into $F$ is strictly differentiable at $\bar{x}$ with derivative $\nabla f(\bar{x})$, that is,

$$
f(x+t v)=f(x)+t \nabla f(\bar{x})(v)+\operatorname{tr}(t, x ; v)
$$

for all $(t, x, v) \in] 0, \varepsilon\left[\times E \times E\right.$ and $\lim _{(x, w) \rightarrow(\bar{x}, v) ; t \downarrow 0} r(t, x ; w)=0$ for all $v \in E$, and if the multifunction $M$ and the point $\bar{y}$ are defined as in Proposition 1.6, then,

$$
T(M ; \bar{x}, \bar{y})(v)=\nabla f(\bar{x})(v)+P
$$

for each $v \in E$.

We shall finish this section by giving an important interpretation of the convexity of $T(M ; \bar{x}, \bar{y})$ in terms of convex processes. These multifunctions verify many important properties (see $[3,8,19,20,32]$ ) as, for example, open mapping and closed graph theorems which are extensions of the usual linear ones.

1.8 Definition. A multifunction $M$ from $E$ into $F$ is said to be convex if its graph, Gr $M$, is convex in $E \times F$.

If $\mathrm{Gr} M$ is a convex cone in $E \times F$ containing the origin one says that $M$ is $a$ convex process.

1.9 Proposition. Let $M$ be a multifunction from $E$ into $F$ with $\bar{y} \in M(\bar{x})$. The multifunction from $E$ into $F$ defined by $v \mapsto T(M ; \bar{x}, \bar{y})(v)$ is a convex process.

Proof. This is a direct consequence of the fact that $T(M ; \bar{x}, \bar{y})$ is a convex cone.

2. Quasi-interiorly tangent cones to multifunctions. Let us begin by broadening to multifunctions the definition of quasi-interiorly tangent cone that we have introduced in [29 and 30] for vector-valued functions.

2.1 Definition. If $M$ is a multifunction from $E$ into $F$ with $\bar{y} \in M(\bar{x})$, the quasi-interiorly tangent cone to the graph of $M$ at $(\bar{x}, \bar{y})$ is the set of $Q(M ; \bar{x}, \bar{y})$ of all $(\bar{v}, \bar{w})$ in $E \times F$ such that for each neighbourhood $W$ of $\bar{w}$ in $F$, there exist a neighbourhood $X$ of $\bar{x}$ in $E$, a neighbourhood $Y$ of $\bar{y}$ in $F$, a real number $\varepsilon>0$ and a neighbourhood $V$ of $\bar{v}$ in $E$ such that

$$
[(x, y)+t(\{v\} \times W)] \cap \operatorname{Gr} M \neq \varnothing
$$

for all $(x, y) \in(X \times Y) \cap \operatorname{Gr} M, t \in] 0, \varepsilon[$ and $v \in V$. 
If $f$ is a mapping from $E$ into $F$ we shall write $Q(f ; \bar{x})$ instead of $Q(f ; \bar{x}, f(\bar{x}))$.

REMARK. If $I(M ; \bar{x}, \bar{y})$ denotes the interiorly tangent cone, that is, the set of all $(v, w) \in E \times F$ for which there exist a neighbourhood $X$ of $\bar{x}$ in $E$, a neighbourhood $Y$ of $\bar{y}$ in $F$, a real number $\varepsilon>0$, a neighbourhood $V$ of $\bar{v}$ in $E$ and a neighbourhood $W$ of $\bar{y}$ in $F$ such that

$$
(X \times Y) \cap \operatorname{Gr} M+] 0, \varepsilon[(V \times W) \subset \mathrm{Gr} M,
$$

it is easy to see that

$$
I(M ; \bar{x}, \bar{y}) \subset Q(M ; \bar{x}, \bar{y}) \subset T(M ; \bar{x}, \bar{y}) .
$$

2.2 Proposition. Let $M$ be a multifunction from $E$ into $F$ with $\bar{y} \in M(\bar{x})$. Then

$$
Q(M ; \bar{x}, \bar{y})+T(M ; \bar{x}, \bar{y}) \subset Q(M ; \bar{x}, \bar{y}) .
$$

Proof. Let $\left(\bar{v}_{1}, \bar{w}_{1}\right) \in Q(M ; \bar{x}, \bar{y})$ and $\left(\bar{v}_{2}, \bar{w}_{2}\right) \in T(M ; \bar{x}, \bar{y})$ and let $W$ be a neighbourhood of $\bar{w}_{1}+\bar{w}_{2}$ in $F$. Choose a neighbourhood $W_{1}$ of $\bar{w}_{1}$ and a neighbourhood $W_{2}$ of $\bar{w}_{2}$ satisfying $W_{1}+W_{2} \subset W$. There exist a neighbourhood $X_{1}$ of $\bar{x}$ in $E$, a neighbourhood $Y_{1}$ of $\bar{y}$ in $F$, a real number $\varepsilon_{1}>0$ and a neighbourhood $V_{1}^{\prime}$ of $\bar{v}_{1}$ in $E$ such that

$$
\left[(x, y)+t\left(\left\{v_{1}\right\} \times W_{1}\right)\right] \cap \operatorname{Gr} M \neq \varnothing
$$

for all $\left.(x, y) \in\left(X_{1} \times Y_{1}\right) \cap \operatorname{Gr} M, t \in\right] 0, \varepsilon_{1}\left[\right.$ and $v_{1} \in V_{1}^{\prime}$. Choose a neighbourhood $Y$ of $\bar{y}$ in $F$, a neighbourhood $W_{2}^{\prime}$ of $\bar{w}_{2}$ in $F$ with $W_{2}^{\prime} \subset W_{2}$, a real number $\varepsilon_{2}>0$, a neighbourhood $X_{2}$ of $\bar{x}$ in $E$, a neighbourhood $V_{2}$ of $\bar{v}_{2}$ in $E$ and a neighbourhood $V_{1}$ of $\bar{v}_{1}$ in $E$ such that

$$
\left.X_{2}+\right] 0, \varepsilon_{2}\left[V_{2} \subset X_{1}, \quad Y_{2}+\right] 0, \varepsilon_{2}\left[W_{2}^{\prime} \subset Y_{1} \text { and } \quad V_{1}+V_{2}-V_{2} \subset V_{1}^{\prime}\right. \text {. }
$$

By Definition 1.2 there exist a neighbourhood $X$ of $\bar{x}$ in $E$ with $X \subset X_{1} \cap X_{2}$, a neighbourhood $Y$ of $\bar{y}$ in $F$ with $Y \subset Y_{1} \cap Y_{2}$ and a real number $\varepsilon>0$ with $\varepsilon<\inf \left(\varepsilon_{1}, \varepsilon_{2}\right)$ such that

$$
\left[(x, y)+t\left(V_{2} \times W_{2}^{\prime}\right)\right] \cap \operatorname{Gr} M \neq \varnothing
$$

for all $(x, y) \in(X \times Y) \cap \operatorname{Gr} M$ and $t \in] 0, \varepsilon$. Consider now a point $(x, y) \in(X$ $\times Y) \cap \operatorname{Gr} M$, a real number $t \in] 0, \varepsilon\left[\right.$ and two elements $v_{1} \in V_{1}$ and $v_{2} \in V_{2}$. By (7) there exist $v_{2}^{\prime} \in V_{2}$ and $w_{2} \in W_{2}^{\prime}$ such that $\left(x+t v_{2}^{\prime}, y+t w_{2}\right) \in \operatorname{Gr} M$. By (6) we have

$$
\left(x+t v_{2}^{\prime}, y+t w_{2}\right) \in X_{1} \times Y_{1} \text { and } v_{1}+v_{2}-v_{2}^{\prime} \in V_{1}^{\prime}
$$

and, hence, there exists by (5) a point $w_{1} \in W_{1}$ such that

$$
\begin{aligned}
\left(x+t v_{1}+t v_{2}, y+t w_{1}+t w_{2}\right) & =\left(x+t v_{2}^{\prime}+t\left(v_{1}+v_{2}-v_{2}^{\prime}\right), y+t w_{1}+t w_{2}\right) \\
& \in \text { Gr } M .
\end{aligned}
$$

Therefore we may write

$$
\left[(x, y)+t\left(\left\{v_{1}+v_{2}\right\} \times W\right)\right] \cap \operatorname{Gr} M \neq \varnothing
$$

and hence $\left(\bar{v}_{1}, \bar{w}_{1}\right)+\left(\bar{v}_{2}, \bar{w}_{2}\right) \in Q(M ; \bar{x}, \bar{y})$. This finishes the proof of the proposition.

Let us give two important consequences of Proposition 2.2. 
2.3 COROllary. Let $M$ be a multifunction from $E$ into $F$ with $\bar{y} \in M(\bar{x})$. Then the quasi-interiorly tangent cone $Q(M ; \bar{x}, \bar{y})$ is a convex cone and for each $v \in E$ the set $Q(M ; \bar{x}, \bar{y})(v)$ is closed in $F$.

Proof. From Definition 2.1 it is readily seen that $Q(M ; \bar{x}, \bar{y})(v)$ is closed in $F$ for each $v \in E$. So by Proposition 2.2 it is enough to prove $Q(M ; \bar{x}, \bar{y})$ is a cone. Consider a real number $\lambda>0$, a point $(\bar{v}, \bar{w})$ in $Q(M ; \bar{x}, \bar{y})$ and a neighbourhood $W$ of $\lambda \bar{w}$ in $F$. The set $W_{0}=\lambda^{-1} W$ is a neighbourhood of $\bar{w}$ in $F$ and hence there exist a neighbourhood $X$ of $\bar{x}$ in $E$, a neighbourhood $Y$ of $\bar{y}$ in $F$, a real number $\varepsilon_{0}>0$ and a neighbourhood $V_{0}$ of $\bar{v}$ in $E$ such that $\left[(x, y)+t\left(\{v\} \times W_{0}\right)\right] \cap \operatorname{Gr} M \neq \varnothing$ for all $(x, y) \in(X \times Y) \cap \mathrm{Gr} M, t \in] 0, \varepsilon_{0}\left[\right.$ and $v \in V_{0}$. Therefore, if we put $V=\lambda V_{0}$ and $\varepsilon=\lambda^{-1} \varepsilon_{0}$, we obtain

$$
[(x, y)+t(\{v\} \times W)] \cap \mathrm{Gr} M \neq \varnothing
$$

for all $(x, y) \in(X \times Y) \cap \operatorname{Gr} M, t \in] 0, \varepsilon[$ and $v \in V$. So $\lambda(\bar{v}, \bar{w}) \in Q(M ; \bar{x}, \bar{y})$ and hence the proof is complete.

2.4 Corollary. Let $M$ be a multifunction from $E$ into $F$ with $\bar{y} \in M(\bar{x})$. If the quasi-interiorly tangent cone $Q(M ; \bar{x}, \bar{y})$ is nonempty, then

$$
T(M ; \bar{x}, \bar{y})=\operatorname{cl}_{E \times F}(Q(M ; \bar{x}, \bar{y})) .
$$

Proof. By (4) it is enough to prove $T(M ; \bar{x}, \bar{y}) \subset \mathrm{cl}_{E \times F}(Q(M ; \bar{x}, \bar{y}))$. Fix a point $(\bar{v}, \bar{w})$ in $Q(M ; \bar{x}, \bar{y})$. For each $(v, w) \in T(M ; \bar{x}, \bar{y})$ and each $\varepsilon>0$ we have by Proposition 2.2,

$$
(v, w)+\varepsilon(\bar{v}, \bar{w}) \in Q(M ; \bar{x}, \bar{y}) .
$$

So, if we write $(v, w)=\lim _{\varepsilon \downarrow}((v, w)+\varepsilon(\bar{v}, \bar{w}))$, we obtain that $(v, w) \in$ $\mathrm{cl}_{E \times F}(Q(M ; \bar{x}, \bar{y}))$ and the proof is complete.

Two other formulations of quasi-interiorly tangent cones can be stated. The first one follows easily from the definition of lower limit (see (1)).

2.5 Proposition. If $M$ is a multifunction from $E$ into $F$ with $\bar{y} \in M(\bar{x})$, then for each $\bar{v} \in E$,

$$
Q(M ; \bar{x}, \bar{y})(\bar{v})=\liminf _{\substack{\left.v \rightarrow \overline{\bar{v}} \\(x, y) \rightarrow M_{(\bar{x}}, \bar{y}\right) \\ t \downarrow 0}} t^{-1}[M(x+t v)-y],
$$

where the notation $(x, y) \rightarrow^{M}(\bar{x}, \bar{y})$ means $(x, y) \rightarrow(\bar{x}, \bar{y})$ with $(x, y) \in \mathrm{Gr} M$.

2.6 Corollary. Let $M$ be a multifunction from $E$ into $F$ with $\bar{y} \in M(\bar{x})$. Then $(\bar{v}, \bar{w})$ is in $Q(M ; \bar{x}, \bar{y})$ if and only if for any net $\left(x_{j}, y_{j}\right)_{j \in J}$ in $\operatorname{Gr} M$ converging to $(\bar{x}, \bar{y})$, any net $\left(t_{j}\right)_{j \in J}$ in $] 0,+\infty\left[\right.$ converging to zero and any net $\left(v_{j}\right)_{j \in J}$ in $E$ converging to $\bar{v}$ there exist subnets $\left(x_{\alpha(i)}, y_{\alpha(i)}\right)_{i \in I},\left(t_{\alpha(i)}\right)_{i \in I},\left(v_{\alpha(i)}\right)_{i \in I}$ and a net $\left(w_{i}\right)_{i \in I}$ converging to $\bar{w}$ such that

$$
y_{\alpha(i)}+t_{\alpha(i)} w_{i} \in M\left(x_{\alpha(i)}+t_{\alpha(i)} v_{\alpha(i)}\right)
$$

for all $i \in I$.

PrOof. This follows from Proposition 1.1. 
We know (see Remark 1 following Definition 2.1) that $I(M ; \bar{x}, \bar{y}) \subset Q(M ; \bar{x}, \bar{y})$. Let us give a condition under which we can say more.

2.7 Proposition. Let $M$ be a multifunction from $E$ into $F$ such that there is an open convex cone $K$ verifying $M(x)+K \subset M(x)$ for all $x \in E$. If $\bar{y} \in M(\bar{x})$ and if $F$ is a locally convex vector space, then for each $v \in E$ :

(i) $I(M ; \bar{x}, \bar{y})(v)=$ int $_{F}[Q(M ; \bar{x}, \bar{y})(v)]$ and

(ii) $Q(M ; \bar{x}, \bar{y})(v)=\operatorname{cl}_{F}[I(M ; \bar{x}, \bar{y})(v)]$.

Proof. Let $\bar{v}$ be a point in $E$. As $I(M ; \bar{x}, \bar{y})$ is open in $E \times F$, the set $I(M ; \bar{x}, \bar{y})(\bar{v})$ is open in $F$ and hence by (4) we have

$$
I(M ; \bar{x}, \bar{y})(\bar{v}) \subset \operatorname{int}_{F}[Q(M ; \bar{x}, \bar{y})(\bar{v})] .
$$

Let us prove the reverse inclusion. Let $\bar{w}$ be a point in int ${ }_{F}[Q(M ; \bar{x}, \bar{y})(\bar{v})]$ and let $\Omega$ be a convex neighbourhood of zero in $F$ such that $\bar{w}+\Omega \subset Q(M ; \bar{x}, \bar{y})(\bar{v})$. Choose two points $a \in(\bar{w}+\Omega) \cap(\bar{w}-K)$ and $b \in(\bar{w}+\Omega) \cap(\bar{w}+K)$. If we put $c=$ $2^{-1}(a+\bar{w})$ and $d=2^{-1}(a+c)$, then we have

$$
d \in \bar{w}+\Omega \text { and } d \in W_{1}:=(a+K) \cap(c-K) .
$$

So $(\bar{v}, d) \in Q(M ; \bar{x}, \bar{y})$ and hence, since $W_{1}$ is open, there exist a neighbourhood $X$ of $\bar{x}$ in $E$, a neighbourhood $Y$ of $\bar{y}$ in $F$, a neighbourhood $V$ of $\bar{v}$ in $E$ and a real number $\varepsilon>0$ such that

$$
\left[(x, y)+t\left(\{v\} \times W_{1}\right)\right] \cap \operatorname{Gr} M \neq \varnothing
$$

for all $(x, y) \in(X \times Y) \cap \operatorname{Gr} M, v \in V$ and $t \in] 0, \varepsilon[$. Put

$$
W=(c+K) \cap(b-K) \text {. }
$$

The set $W$ is a neighbourhood of $\bar{w}$ in $F$ and for each $w \in W$, each $(x, y) \in(X \times$ $Y) \cap \operatorname{Gr} M$, each $v \in V$ and each $t \in] 0, \varepsilon$ [ there exist, by (8), an element $w_{1} \in W_{1}$ such that $y+t w_{1} \in M(x+t v)$ and hence, since $w-w_{1} \in K$,

$$
y+t w=y+t w_{1}+t\left(w-w_{1}\right) \in M(x+t v)+K \subset M(x+t v) .
$$

This shows that

$$
\text { int }_{F}[Q(M ; \bar{x}, \bar{y})(\bar{v})] \subset I(M ; \bar{x}, \bar{y})(\bar{v}) .
$$

Let us prove (ii). It is not difficult to see that

$$
K+Q(M ; \bar{x}, \bar{y})(\bar{v}) \subset Q(M ; \bar{x}, \bar{y})(\bar{v})
$$

and, hence, by (i), int $[Q(M ; \bar{x}, \bar{y})(\bar{v})] \neq \varnothing$ if and only if $Q(M ; \bar{x}, \bar{y})(\bar{v}) \neq \varnothing$. So, since $Q(M ; \bar{x}, \bar{y})(\bar{v})$ is a closed convex set we conclude by (i) that

$$
Q(M ; \bar{x}, \bar{y})(\bar{v})=\operatorname{cl}_{F}[I(M ; \bar{x}, \bar{y})(\bar{v})] .
$$

In the sequel of this section we shall consider the two important classes of lipschitzian multifunctions and of convex multifunctions.

Let us begin with lipschitzian multifunctions.

2.8 Definition. We shall say that a multifunction $M$ from $E$ into $F$ is lipschitzian at a point $\bar{x} \in \operatorname{dom} M$ in the direction $\bar{v}$ if for each neighbourhood $U$ of zero in $F$ there exist a neighbourhood $X$ of $\bar{x}$ in $E$, a neighbourhood $V$ of $\bar{v}$ in $E$ and a real 
number $\varepsilon>0$ such that

$$
M\left(x+t v_{1}\right) \subset M\left(x+t v_{2}\right)+t U
$$

for all $x \in X, v_{1} \in V, v_{2} \in V$ and $\left.\left.t \in\right] 0, \varepsilon\right]$.

REMARK. If $F$ is a normed vector space, it is not difficult to see that $M$ is lipschitzian at $\bar{x}$ in the direction $\bar{v}$ if and only if

$$
\lim _{\substack{\left(x, v_{1}, v_{2}\right) \rightarrow(\bar{x}, \bar{v}, \bar{v}) \\ t \downarrow 0}} t^{-1} \operatorname{dist}\left(M\left(x+t v_{1}\right), M\left(x+t v_{2}\right)\right)=0,
$$

where dist denotes the Hausdorff distance.

2.9 Proposition. Let $M$ be a multifunction from $E$ into $F$ which is lipschitzian at $\bar{x} \in \operatorname{dom} M$ in the direction $\bar{v}$. If $\bar{y} \in M(\bar{x})$, then

$$
T(M ; \bar{x}, \bar{y})(\bar{v})=Q(M ; \bar{x}, \bar{y})(\bar{v}) .
$$

Proof. Because of (4), it suffices to prove that $T(M ; \bar{x}, \bar{y})(\bar{v}) \subset Q(M ; \bar{x}, \bar{y})(\bar{v})$. Let $\bar{w}$ be a point in $T(M ; \bar{x}, \bar{y})(\bar{v})$ and let $W$ be a neighbourhood of $\bar{w}$ in $F$. Select a circled neighbourhood $U$ of zero in $F$ and a neighbourhood $W_{1}$ of $\bar{w}$ in $F$ such that $W_{1}+U \subset W$. By Definition 2.8 there exist a neighbourhood $X_{1}$ of $\bar{x}$, a neighbourhood $V$ of $\bar{v}$ in $E$ and a number $\varepsilon_{1}>0$ such that

$$
M\left(x+t v_{1}\right) \subset M\left(x+t v_{2}\right)+t U
$$

for all $x \in X_{1}, v_{1} \in V, v_{2} \in V$ and $\left.\left.t \in\right] 0, \varepsilon_{1}\right]$. Choose, by Definition 1.2, a neighbourhood $X_{2}$ of $\bar{x}$ in $E$, a neighbourhood $Y$ of $\bar{y}$ in $F$ and a real number $\varepsilon_{2}>0$ such that

$$
\left[(x, y)+t\left(V \times W_{1}\right)\right] \cap \operatorname{Gr} M \neq \varnothing
$$

for all $(x, y) \in(X \times Y) \cap \operatorname{Gr} M$ and $\left.t \in] 0, \varepsilon_{2}\right]$. Put $X=X_{1} \cap X_{2}$ and $\varepsilon=$ $\inf \left(\varepsilon_{1}, \varepsilon_{2}\right)$ and consider a point $(x, y) \in(X \times Y) \cap \operatorname{Gr} M$, a point $v \in V$ and a real number $t \in] 0, \varepsilon]$. By (10) there exists $\left(v_{1}, w_{1}\right) \in V \times W_{1}$ such that $y+t w_{1} \in$ $M\left(x+t v_{1}\right)$. Therefore it follows from (9) that $y+t w_{1} \in M(x+t v)+t U$ and, hence, there exists $u \in U$ such that

$$
y+t\left(w_{1}+u\right) \in M(x+t v) \text { and } w_{1}+u \in W .
$$

This shows that

$$
[(x, y)+t(\{v\} \times W)] \cap \operatorname{Gr} M \neq \varnothing,
$$

and the proof is complete.

2.10 COROLlary. Let $M$ be a multifunction from $E$ into $F$ with $\bar{y} \in M(\bar{x})$. If there exists a neighbourhood $Y$ of $\bar{y}$ in $F$ such that the multifunction $N$ defined by $N(x)=$ $M(x) \cap Y$ for each $x \in E$ is lipschitzian at $\bar{x}$ in the direction $\bar{v}$, then $T(M ; \bar{x}, \bar{y})(\bar{v})=$ $Q(M ; \bar{x}, \bar{y})(\bar{v})$.

Proof. We have $\operatorname{Gr} N=(E \times Y) \cap \operatorname{Gr} M$, and, hence, by Definitions 1.2 and 2.1 one easily sees $T(N ; \bar{x}, \bar{y})=T(M ; \bar{x}, \bar{y})$ and $Q(N ; \bar{x}, \bar{y})=Q(M ; \bar{x}, \bar{y})$. So it suffices to apply Proposition 2.9 to the multifunction $N$. 
2.11 Corollary. Let $f$ be a mapping from $E$ into $F$ and let $\bar{v}$ be a point in $E$ such that

$$
\lim _{\substack{t \downarrow 0 \\\left(x, v_{1}, v_{2}\right) \rightarrow(\bar{x}, \bar{v}, \bar{v})}} t^{-1}\left[f\left(x+t v_{1}\right)-f\left(x+t v_{2}\right)\right]=0,
$$

and let $M$ be a multifunction defined by

$$
M(x)=f(x)+K \text { for every } x \in E,
$$

where $K$ is a nonempty subset of $F$. Then for each $\bar{y} \in M(\bar{x})$ we have $T(M ; \bar{x}, \bar{y})(\bar{v})=$ $Q(M ; \bar{x}, \bar{y})(\bar{v})$.

Proof. If we put

$$
r\left(t, x ; v_{1}, v_{2}\right)=t^{-1}\left[f\left(x+t v_{1}\right)-f\left(x+t v_{2}\right)\right],
$$

it is not difficult to see that

$$
M\left(x+t v_{1}\right) \subset M\left(x+t v_{2}\right)+\operatorname{tr}\left(t, x ; v_{1}, v_{2}\right)
$$

and, hence, the result is a consequence of Proposition 2.9.

2.12 Corollary [29, Proposition 3-10]. Let $f$ be a mapping from $E$ into $F$ which is strictly compactly lipschitzian at $\bar{x} \in E$ and let $M$ be the multifunction defined as in Corollary 2.11. Then for $\bar{y} \in M(\bar{x})$ we have $T(M ; \bar{x}, \bar{y})=Q(M ; \bar{x}, \bar{y})$.

Proof. This is a direct consequence of Corollary 2.11 and of the remarks following the definition of a strictly compactly lipschitzian mapping.

Before stating our first two results (Lemma 2.19 and Proposition 2.20) about the relationships between tangent cones and quasi-interiorly tangent cones to convex multifunctions, we shall need some notions and lemmas.

2.13 Definition. A multifunction $M$ from a topological space $X$ into a topological space $Y$ is lower semicontinuous at a point $\bar{x} \in X$ if for each open subset $\Omega$ in $Y$ such that $M(\bar{x}) \cap \Omega \neq \varnothing$ there exists a neighbourhood $V$ of $\bar{x}$ in $E$ such that $M(x) \cap \Omega$ $\neq \varnothing$ for each $x \in V$. In other words $\liminf _{x \rightarrow \bar{x}} M(x) \supset M(\bar{x})$.

If $M$ is lower semicontinuous at each point of a subset $D$ of $X$, one says $M$ is lower semicontinuous on $D$.

If $Z$ is a subset of $X$ with $\bar{x} \in Z$, one says that $M$ is lower semicontinuous at $\bar{x}$ relative to $Z$ if the restriction of $M$ to $Z$ is lower semicontinuous at $\bar{x}$ wih respect to the topology induced on $Z$ by that of $X$.

2.14 LEMMA. Let $M$ be a multifunction from a topological space $X$ into a topological vector space $Y$ which is lower semicontinuous at $\bar{x} \in \operatorname{dom} M$ and let $f$ be a mapping from $\operatorname{dom} f \subset X$ into $Y$. If $\bar{x} \in \operatorname{int} \operatorname{dom} f$ and if $f$ is continuous at $\bar{x}$, then the multifunction $M+f$ defined by $(M+f)(x)=M(x)+f(x)$ is lower semicontinuous at $\bar{x}$.

Proof. Let $\Omega$ be an open subset of $Y$ such that $(M(\bar{x})+f(\bar{x})) \cap \Omega \neq \varnothing$. Consider a point $b \in(M(\bar{x})+f(\bar{x})) \cap \Omega$ and an open circled neighbourhood $W$ of zero in $Y$ such that $b+W+W \subset \Omega$. Then $M(\bar{x}) \cap(b-f(\bar{x})+W) \neq \varnothing$, and hence there exists a neighbourhood $V_{1}$ of $\bar{x}$ in $X$ such that

$$
M(x) \cap(b-f(\bar{x})+W) \neq \varnothing \quad \text { for all } x \in V_{1} .
$$


Moreover, since $\bar{x} \in \operatorname{int} \operatorname{dom} f$ and since $f$ is continuous at $\bar{x}$, there exists a neighbourhood $V_{2}$ of $\bar{x}$ in $X$ such that

$$
f(x) \in f(\bar{x})+W \text { for all } x \in V_{2} .
$$

So if we put $V=V_{1} \cap V_{2}$, relations (11) and (12) imply that $(M(x)+f(x)) \cap \Omega \neq$ $\varnothing$ for all $x \in V$, and the proof is complete.

REMARK. More generally, the above proof shows that the sum of two lower semicontinuous multifunctions is lower semicontinuous.

2.15 LeMma. Let $M$ be a convex multifunction from $E$ into $F$ whose graph has a nonempty interior. Then $(\bar{x}, \bar{y}) \in \operatorname{int} \operatorname{Gr} M$ if and only if $\bar{x} \in \operatorname{int} \operatorname{dom} M$ and $\bar{y} \in$ int $M(\bar{x})$.

Proof. Note first that

$$
\operatorname{int}\left(\operatorname{proj}_{E}(\operatorname{Gr} M)\right)=\operatorname{proj}_{E}(\operatorname{int}(\operatorname{Gr} M)) \text {. }
$$

Indeed let $x$ be any point in $\operatorname{int}\left(\operatorname{proj}_{E}(\operatorname{Gr} M)\right)$. Fix a point $(a, b)$ in $\operatorname{int}(\mathrm{Gr} M)$. There exists a real number $s>1$ such that $s x+s\left(s^{-1}-1\right) a \in \operatorname{proj}_{E}(\mathrm{Gr} M)$. Choose $y \in M\left(s x+s\left(s^{-1}-1\right) a\right)$. Then it follows from the convexity of $\operatorname{Gr} M$ that $\left(x, s^{-1} y\right.$ $\left.+\left(1-s^{-1}\right) b\right) \in \operatorname{int} \operatorname{Gr} M$ and hence $x \in \operatorname{proj}_{E}(\operatorname{int} \operatorname{Gr} M)$. So (13) is verified, for the inclusion proj(int $\operatorname{Gr} M) \subset \operatorname{int}(\operatorname{proj} \operatorname{Gr} M)$ is obvious. Consider now a point $(\bar{x}, \bar{y})$ such that $\bar{x} \in$ int $\operatorname{dom} M$ and $\bar{y} \in$ int $M(\bar{x})$. By (13) there exists $z$ in $F$ such that $(\bar{x}, z) \in \operatorname{int} \operatorname{Gr} M$. Choose a real number $t>1$ such that $t \bar{y}+t\left(t^{-1}-1\right) z \in$ $M(\bar{x})$. Then we may conclude that

$$
(\bar{x}, \bar{y})=t^{-1}\left(\bar{x}, t \bar{y}+t\left(t^{-1}-1\right) z\right)+\left(1-t^{-1}\right)(\bar{x}, z) \in \operatorname{int} \text { Gr } M,
$$

for $\mathrm{Gr} M$ convex. Since the reverse implication is obvious, the proof is complete.

2.16 LeMMA. Let $M$ be a convex multifunction from $E$ into $F$ whose graph has $a$ nonempty interior. Then $M$ is lower semicontinuous on int dom $M$.

Proof. Let $\bar{x}$ be a point in int $\operatorname{dom} M$ and let $\Omega$ be an open subset in $F$ such that $M(\bar{x}) \cap \Omega \neq \varnothing$. Since $\operatorname{Gr} M$ is a convex set with nonempty interior and since $\bar{x} \in$ int dom $M$, it follows from (13) that int $M(\bar{x}) \neq \varnothing$ and hence $\Omega \cap$ int $M(\bar{x}) \neq$ $\varnothing$. Therefore, we may choose $\bar{y} \in \Omega \cap$ int $M(\bar{x})$ and by Lemma 2.15 we have $(\bar{x}, \bar{y}) \in \operatorname{int}(\operatorname{Gr} M)$. So, there exists a neighbourhood $X$ of $\bar{x}$ in $E$ such that $X \times\{\bar{y}\} \subset \mathrm{Gr} M$ or, in other words, $\bar{y} \in M(x)$ for all $x \in X$ and hence we may conclude that $\Omega \cap M(x) \neq \varnothing$ for all $x \in X$.

REMARK. If $E$ and $F$ are finite dimensional, then the assumption that $\operatorname{Gr} M$ has a nonempty interior can be supressed, for it suffices to replace in the above proof $\operatorname{int}(\mathrm{Gr} M)$ by $\operatorname{ri}(\mathrm{Gr} M)$ and int $M(\bar{x})$ by ri $M(\bar{x})$. Here the notation ri means the relative interior (see [21]).

2.17 Definition. If $C$ is a nonempty convex subset of $E$ with $\bar{x} \in C$, the radial tangent cone to $C$ at $\bar{x}$ is the set

$$
R(C ; \bar{x})=] 0,+\infty[(C-\bar{x}) .
$$

The proof of the following proposition given in [23] (see Theorem 1) makes use of the assumption that $E$ is locally convex. Actually the result holds for any topological vector space, and this will be used in Proposition 2.20 and in other ones. 
2.18 Proposition. If $C$ is a convex subset in $E$ with $\bar{x} \in C$, then

$$
T(C ; \bar{x})=\operatorname{cl}_{E}[R(C ; \bar{x})] .
$$

Proof. Let $\bar{v} \in T(C ; \bar{x})$ and let $V$ be a neighbourhood of $\bar{v}$ in $E$. Definition 1.2 implies there exist $\varepsilon>0$ and $v \in V$ such that $\bar{x}+\varepsilon v \in C$ and hence $v \in V \cap$ $R(C ; \bar{x})$. So $\bar{v} \in \mathrm{cl}_{E}[R(C ; \bar{x})]$ and hence $T(C ; \bar{x}) \subset \mathrm{cl}_{E}[R(C ; \bar{x})]$. To prove the reverse inclusion, it is enough to show that $R(C ; \bar{x}) \subset T(C ; \bar{x})$, for $T(C ; \bar{x})$ is closed in $E$. Let $\bar{v} \in R(C ; \bar{x})$ and let $V$ be a neighbourhood of $\bar{v}$ in $E$. By Definition 2.17 there exist a real number $\varepsilon>0$, an element $\bar{y} \in C$ such that $\bar{y}=\bar{x}+\varepsilon \bar{v}$. Consider a circled neighbourhood $W$ of zero in $E$ such that $\bar{v}+W \subset V$ and put $X=\bar{x}+\varepsilon W$. For each $x=\bar{x}+\varepsilon w \in X \cap C$, we may write

$$
\bar{v}-w \in V \text { and } \bar{y}=\bar{x}+\varepsilon \bar{v}=x+\varepsilon(\bar{v}-w) \in C
$$

and hence $x+t(\bar{v}-w) \in C$ for all $t \in] 0, \varepsilon$, for $C$ is convex and $x \in C$. Therefore, $(x+t V) \cap C \neq \varnothing$ for all $x \in X \cap C$ and $t \in] 0, \varepsilon[$ and hence $\bar{v} \in T(C ; \bar{x})$.

2.19 LemMa. Let $M$ be a convex multifunction from $E$ into $F$ with $\bar{y} \in M(\bar{x})$. Let $\bar{v}$ be a point in $E$ for which $T(M ; \bar{x}, \bar{y})(\bar{v})=\mathrm{cl}_{F}[R(M ; \bar{x}, \bar{y})(\bar{v})]$ and for which there exists a real number $\alpha>0$ such that the multifunction $M$ is lower semicontinuous on $\bar{x}+] 0, \alpha] \bar{v} \subset \operatorname{dom} M$. Then $T(M ; \bar{x}, \bar{y})(\bar{v})=Q(M ; \bar{x}, \bar{y})(\bar{v})$.

Proof. Since $Q(M ; \bar{x}, \bar{y})(\bar{v})$ is closed (see Proposition 2.3), it is enough to prove that $R(M ; \bar{x}, \bar{y})(\bar{v}) \subset Q(M ; \bar{x}, \bar{y})(\bar{v})$, for by $(4), Q(M ; \bar{x}, \bar{y})(\bar{v}) \subset T(M ; \bar{x}, \bar{y})(\bar{v})$. Let $\bar{w}$ be a point in $R(M ; \bar{x}, \bar{y})(\bar{v})$ and let $W$ be an open neighbourhood of $\bar{w}$ in $F$. There exists by Definition 2.17 a positive number $s \leqslant \alpha$ such that

$$
\bar{y}+s \bar{w} \in M(\bar{x}+s \bar{v})
$$

and hence $W \cap s^{-1}[M(\bar{x}+s \bar{v})-\bar{y}] \neq \varnothing$. Since $M$ is lower semicontinuous at $\bar{x}+s \bar{v}$, it follows from Lemma 2.14 that there exists a neighbourhood $X$ of $\bar{x}$ in $E$, a neighbourhood $V$ of $\bar{v}$ in $E$ and a neighbourhood $Y$ of $\bar{y}$ in $F$ such that $W \cap$ $s^{-1}[M(x+s v)-y] \neq \varnothing$ for all $x \in X, y \in Y$ and $v \in V$. So if $(x, y) \in(X \times Y)$ $\cap \operatorname{Gr} M$ and $v \in V$ there exists $w \in W$ such that $(x, y)+s(v, w) \in \operatorname{Gr} M$ and hence, since $\operatorname{Gr} M$ is convex, we have $(x, y)+t(v, w) \in \mathrm{Gr} M$ for all $t \in] 0, s]$. Therefore we may write

$$
[(x, y)+t(\{v\} \times W)] \cap \operatorname{Gr} M \neq \varnothing
$$

for all $(x, y) \in(X \times Y) \cap \operatorname{Gr} M, v \in V$ and $t \in] 0, s]$, that is, $\bar{w} \in Q(M ; \bar{x}, \bar{y})(\bar{v})$.

REMARK. The above proof also shows that, if $M$ is a convex multifunction which is lower semicontinuous on $\bar{x}+] 0, \alpha] \bar{v}$ (for some $\alpha>0$ ), then $R(M ; \bar{x}, \bar{y})(\bar{v}) \subset$ $Q(M ; \bar{x}, \bar{y})(\bar{v})$ and hence $Q(M ; \bar{x}, \bar{y})(\bar{v}) \neq \varnothing$, for Definition 2.15 implies that $R(M ; \bar{x}, \bar{y})(\bar{v}) \neq \varnothing$ for such $\bar{v}$.

We can now give our first principal result about the relationships between tangent cones and quasi-interiorly tangent cones to convex multifunctions.

2.20 Proposition. Let $M$ be a convex multifunction from $E$ into $F$ whose graph has a nonempty interior in $E \times F$. If $\bar{y} \in M(\bar{x})$ and if $\bar{v}$ is a point for which there exists a real number $\alpha>0$ such that $\bar{x}+\alpha \bar{v} \in \operatorname{int} \operatorname{dom} M$, then $T(M ; \bar{x}, \bar{y})(\bar{v})=$ $Q(M ; \bar{x}, \bar{y})(\bar{v})$. 
Proof. According to Lemmas 2.16 and 2.19 it is enough to prove that

$$
T(M ; \bar{x}, \bar{y})(\bar{v})=\mathrm{cl}_{F}[R(M ; \bar{x}, \bar{y})(\bar{v})] .
$$

Let $\bar{w}$ be a point in $T(M ; \bar{x}, \bar{y})(\bar{v})$. Definition 2.17 implies that $R(M ; \bar{x}, \bar{y})$ has a nonempty interior in $E \times F$. Moreover since $\bar{x}+\alpha \bar{v} \in$ int dom $M$, there exists a neighbourhood $V$ of $\bar{v}$ in $E$ such that $\bar{x}+\alpha V \subset \operatorname{dom} M$. Therefore, it is not difficult to see that $V \subset \operatorname{proj}_{E}[R(M ; \bar{x}, \bar{y})]$ and hence, since $R(M ; \bar{x}, \bar{y})$ is a convex set with nonempty interior, there exists by (13) a point $w \in F$ such that $(\bar{v}, w) \in$ int $_{E \times F}[R(M ; \bar{x}, \bar{y})]$. So, for each $\left.\left.\varepsilon \in\right] 0,1\right],(\bar{v},(1-\varepsilon) \bar{w}+\varepsilon w) \in R(M ; \bar{x}, \bar{y})$, for by Proposition $2.18,(\bar{v}, \bar{w}) \in \mathrm{cl}_{E \times F}[R(M ; \bar{x}, \bar{y})]$, and hence

$$
\bar{w}=\lim _{\varepsilon \downarrow 0}((1-\varepsilon) \bar{w}+\varepsilon w) \in \operatorname{cl}_{F} R(M ; \bar{x}, \bar{y})(\bar{v})
$$

2.21 Corollary. Let $M$ be a convex multifunction from $E$ into $F$ whose graph has a nonempty interior in $E \times F$. If $\bar{y} \in M(\bar{x})$ and if $\bar{x} \in$ int $\operatorname{dom} M$, then $T(M ; \bar{x}, \bar{y})(v)$ $=Q(M ; \bar{x}, \bar{y})(v)$ for all $v \in E$.

Proof. For each $v \in E$ there exists a real number $\alpha>0$ such that $\bar{x}+\alpha v \in$ int dom $M$ and hence the result is a direct consequence of Proposition 2.20.

In the finite-dimensional case we can obtain the result of Proposition 2.20 under weaker assumptions.

2.22 Proposition. If $E$ and $F$ are finite dimensional topological vector spaces, if $M$ is a convex multifunction from $E$ into $F$ with $\bar{y} \in M(\bar{x})$ and if $\bar{v}$ is a point for which there exists a real number $\alpha>0$ such that $\bar{x}+\alpha \bar{v} \in \operatorname{int} \operatorname{dom} M$, then $T(M ; \bar{x}, \bar{y})(\bar{v})$ $=Q(M ; \bar{x}, \bar{y})(\bar{v})$.

Proof. Let $\bar{w} \in T(M ; \bar{x}, \bar{y})(\bar{v})$. As in the first part of the proof of Proposition 2.20 , one can show that

$$
\bar{v} \in \operatorname{int}_{E}\left(\operatorname{proj}_{E}(R(M ; \bar{x}, \bar{y}))\right) .
$$

Choose a point $w \in \operatorname{ri}(R(M ; \bar{x}, \bar{y})(\bar{v}))$ (relative interior). Then Theorems 6.8 and 6.1 of [21] and our Proposition 2.18 imply that

$$
(\bar{v}, w) \in \operatorname{ri} R(M ; \bar{x}, \bar{y}) \text { and }(\bar{v},(1-\varepsilon) \bar{w}+\varepsilon w) \in R(M ; \bar{x}, \bar{y})
$$

for all $\varepsilon \in] 0,1]$ and hence we may write

$$
\bar{w}=\lim _{\varepsilon \downarrow 0}((1-\varepsilon) \bar{w}+\varepsilon w) \in \operatorname{cl}_{F}[R(M ; \bar{x}, \bar{y})(\bar{v})] .
$$

Therefore, according to Lemma 2.19 and the remark following Lemma 2.16 we have the desired result.

The second principal result about quasi-interiorly tangent cones to convex multifunctions will be stated in the context of normed vector spaces.

2.23 Proposition. If $E$ and $F$ are two normed vector spaces, if $M$ is a convex multifunction from $E$ into $F$ with $\bar{y} \in M(\bar{x})$ and if $\bar{x} \in \operatorname{int} \operatorname{dom} M$, then $T(M ; \bar{x}, \bar{y})(v)=Q(M ; \bar{x}, \bar{y})(v)$ for all $v \in E$. 
Proof. Let $Y$ be the closed unit ball about $\bar{y}$. Consider the multifunction $N$ from $E$ into $F$ whose graph is $\mathrm{Gr} N=(E \times Y) \cap \mathrm{Gr} M$. The multifunction $N$ is convex and takes its values in a bounded subset of $F$. So, by Corollary 5.2 of [16] it is lipschitzian on a neighbourhood of $\bar{x}$ with respect to the Hausdorff metric and hence the proposition follows from Corollary 2.10 as is easily seen.

We shall finish this section by stating two results which are conneced with the tangential condition $\left(T_{2}\right)$ that we have considered in [29].

2.24 Proposition. Let $M$ be a convex multifunction from $E$ into $F$ and $\bar{y} \in M(\bar{x})$. If there exist a point $\bar{v} \in E$ and a number $\alpha>0$ such that $M$ is lower semicontinuous on $\bar{x}+10, \alpha] \bar{v}$, then $T(M ; \bar{x}, \bar{y})=\operatorname{cl}_{E \times F}[Q(M ; \bar{x}, \bar{y})]$.

Proof. This follows from Corollary 2.4 and from the remark following Lemma 2.19 .

2.25 Corollary (COMPare with Proposition 3-11 of [29]). Let $K$ be a convex cone in $F$ and let $f$ be a mapping from dom $f \subset E$ into $F$ which is $K$-convex, that is, $\operatorname{dom} f$ is convex and

$$
f\left(t x_{1}+(1-t) x_{2}\right) \in t f\left(x_{1}\right)+(1-t) f\left(x_{2}\right)-K
$$

for all $t \in[0,1]$ and $x_{1}, x_{2} \in \operatorname{dom} f$. Assume that there exist a point $\bar{v} \in E$ and a real number $\alpha>0$ such that $f$ is continuous on $\bar{x}+] 0, \alpha] \bar{v}$. Then the multifunction $M$ defined by

$$
M(x)=f(x)+K \quad \text { if } x \in \operatorname{dom} f \text { and } M(x)=\varnothing \quad \text { if } x \notin \operatorname{dom} f
$$

verifies the equality

$$
T(M ; \bar{x}, \bar{y})=\mathrm{cl}_{E \times F}[Q(M ; \bar{x}, \bar{y})] .
$$

Proof. This follows from Proposition 2.24 and Lemma 2.14.

3. Sum of two multifunctions. The case of the intersection of two multifunctions can be seen as a particular case of Corollary 3 of [24], for the graph of the intersection of two multifunctions is the intersection of the graphs. So we shall concentrate our attention on the sum of two multifunctions and the composition of a multifunction with a differentiable mapping.

The following definition will be very useful in the sequel.

3.1 Definition. Let $M_{1}$ and $M_{2}$ be two multifunctions from $E$ into $F$ and let $\bar{y}_{1} \in M_{1}(\bar{x})$ and $\bar{y}_{2} \in M_{2}(\bar{x})$. We shall say that $M_{1}$ and $M_{2}$ are additively separate at $\left(\bar{x} ; \bar{y}_{1}, \bar{y}_{2}\right)$ if for every neighbourhood $Y_{1}$ of $\bar{y}_{1}$ and every neighbourhood $Y_{2}$ of $\bar{y}_{2}$ in $F$ there exist a neighbourhood $Y$ of $\bar{y}_{1}+\bar{y}_{2}$ in $F$ and a neighbourhood $X$ of $\bar{x}$ in $E$ such that

$$
\left(M_{1}(x)+M_{2}(x)\right) \cap Y \subset Y_{1} \cap M_{1}(x)+Y_{2} \cap M_{2}(x)
$$

for all $x \in X$.

Let us give two important examples of additively separate multifunctions. 
3.2 Proposition. Let $f$ be a mapping from $\operatorname{dom} f \subset E$ into $F$ and let $M$ be a multifunction from $E$ into $F$. Let $K$ be a subset of $F$ with $0 \in K$ and let $N$ be the multifunction from $E$ into $F$ defined by

$$
N(x)=f(x)+K \quad \text { if } x \in \operatorname{dom} f \text { and } \quad N(x)=\varnothing \quad \text { if } x \notin \operatorname{dom} f .
$$

If $\bar{x} \in \operatorname{dom} f \cap \operatorname{dom} M$, if $f$ is continuous at $\bar{x}$ relative to $\operatorname{dom} f \cap \operatorname{dom} M$ and if $K+M(x) \subset M(x)$ for all $x \in E$, then for any $\bar{y} \in M(\bar{x})$ the multifunctions $M$ and $N$ are additively separate at $(\bar{x} ; \bar{y}, f(\bar{x}))$.

Proof. Let $Y_{1}$ be a neighbourhood of $\bar{y}$ and let $Y_{2}$ be a neighbourhood of $f(\bar{x})$ in $F$. Select a circled neighbourhood $W$ of zero in $F$ such that $W+W \subset\left(Y_{1}-\bar{y}\right) \cap$ $\left(Y_{2}-f(\bar{x})\right)$ and a neighbourhood $X$ of $\bar{x}$ in $E$ such that $f(X \cap \operatorname{dom} f \cap \operatorname{dom} M) \subset$ $f(\bar{x})+W$. Put $Y=\bar{y}+f(\bar{x})+W$. For each $x \in X \cap \operatorname{dom} M \cap \operatorname{dom} N$ and each $y \in(M(x)+N(x)) \cap Y$ we have

$$
f(x) \in f(\bar{x})+W \subset Y_{2}, \quad y-f(x) \in \bar{y}+W+W \subset Y_{1}
$$

and

$$
y-f(x) \in M(x)+K \subset M(x)
$$

and hence

$$
(M(x)+N(x)) \cap Y \subset Y_{1} \cap M(x)+Y_{2} \cap N(x) .
$$

As a direct consequence of the above proposition, we have the following result.

3.3 CoRollary. Let $f_{i}, i=1,2$, be two mappings from $\operatorname{dom} f_{i} \subset E$ into $F$ and let $K$ be a convex cone containing the origin of $F$. If $f_{1}$ is continuous at $\bar{x} \in \operatorname{dom} f_{1} \cap \operatorname{dom} f_{2}$ relative to dom $f_{1} \cap \operatorname{dom} f_{2}$, then the multifunctions $M_{1}$ and $M_{2}$ defined by

$$
M_{i}(x)=f_{i}(x)+K \quad \text { if } x \in \operatorname{dom} f_{i} \text { and } M_{i}(x)=\varnothing \quad \text { if } x \notin \operatorname{dom} f_{i}
$$

are additively separate at $\left(\bar{x} ; f_{1}(\bar{x}), f_{2}(\bar{x})\right)$.

Let us recall the following extension (see [17]) of the notion of lower semicontinuity to vector-valued functions.

3.4 Definition. Let $K$ be a convex cone containing the origin of $F$ and let $f$ be a mapping from dom $f \subset E$ into $F$. We shall say that $f$ is $K$-lower semicontinuous at $\bar{x} \in \operatorname{dom} f$ relative to a subset $D$ containing $\bar{x}$ if for every neighbourhood $W$ of zero in $F$ there is a neighbourhood $X$ of $\bar{x}$ in $E$ such that $f(X \cap D \cap \operatorname{dom} f) \subset f(\bar{x})+$ $W+K$.

In the next proposition we shall use this notion under the assumption that $K$ is a normal cone in $F$ (see [18]), that is, there is a neighbourhood basis $\{W\}_{W}$ of zero such that $(W+K) \cap(W-K)=W$. Such neighbourhoods are called $K$-normal.

3.5 Proposition. Let $K$ be a normal convex cone in $F$ containing the origin and let $f_{i}, i=1,2$, be two mappings from $\operatorname{dom} f_{i} \subset E$ into $F$ which are $K$-lower semicontinuous at $\bar{x} \in \operatorname{dom} f_{1} \cap \operatorname{dom} f_{2}$ relative to $\operatorname{dom} f_{1} \cap \operatorname{dom} f_{2}$. Then the multifunctions $M_{i}$, $i=1,2$, from $E$ into $F$ defined by

$$
M_{i}(x)=f_{i}(x)+K \quad \text { if } x \in \operatorname{dom} f_{i} \quad \text { and } \quad M_{i}(x)=\varnothing \quad \text { if } x \notin \operatorname{dom} f_{i}
$$

are additively separate at $\left(\bar{x} ; f_{1}(\bar{x}), f_{2}(\bar{x})\right)$. 
Proof. Let $Y_{i}, i=1,2$, be two neighbourhoods of $f_{i}(\bar{x})$. Let $W, W^{\prime}$ and $W^{\prime \prime}$ be three normal neighbourhoods of zero in $F$ such that $W^{\prime \prime}-W^{\prime \prime} \subset\left(Y_{1}-f_{1}(\bar{x})\right) \cap$ $\left(Y_{2}-f_{2}(\bar{x})\right), W^{\prime}-W^{\prime} \subset W^{\prime \prime}$ and $W+W \subset W^{\prime}$. Put $Y=f_{1}(\bar{x})+f_{2}(\bar{x})+W$ and choose a neighbourhood $X$ of $\bar{x}$ in $E$ such that

$$
f_{i}\left(X \cap \operatorname{dom} f_{1} \cap \operatorname{dom} f_{2}\right) \subset f_{i}(\bar{x})+W+K .
$$

Consider a point $x \in X \cap \operatorname{dom} f_{1} \cap \operatorname{dom} f_{2}$ and a point $y \in Y \cap\left(M_{1}(x)+M_{2}(x)\right)$. We have

$$
f_{1}(x)+f_{2}(x) \in y-K \subset f_{1}(\bar{x})+f_{2}(\bar{x})+W-K
$$

and hence, because of (14),

$$
\begin{aligned}
f_{1}(x)+f_{2}(x) & \in f_{1}(\bar{x})+f_{2}(\bar{x})+\left(W^{\prime}+K\right) \cap\left(W^{\prime}-K\right) \\
& =f_{1}(\bar{x})+f_{2}(\bar{x})+W^{\prime} .
\end{aligned}
$$

Therefore, we may write, thanks to (14) and (15),

$$
f_{2}(x) \in f_{2}(\bar{x})+W^{\prime}-W^{\prime}-K
$$

and, hence again by (14),

$$
f_{2}(x) \in f_{2}(\bar{x})+\left(W^{\prime \prime}+K\right) \cap\left(W^{\prime \prime}-K\right)=f_{2}(\bar{x})+W^{\prime \prime} \subset Y_{2} \text {. }
$$

Moreover, $y-f_{2}(x) \in f_{1}(x)+K+K=M_{1}(x)$ and the definition of $Y$ and (16) imply that

$$
y-f_{2}(x) \in f_{1}(\bar{x})+f_{2}(\bar{x})+W-f_{2}(x) \subset f_{1}(\bar{x})+W-W^{\prime \prime} \subset Y_{1} .
$$

Therefore,

$$
y=\left(y-f_{2}(x)\right)+f_{2}(x) \in Y_{1} \cap M_{1}(x)+Y_{2} \cap M_{2}(x),
$$

and hence the proof is complete.

Recall that the sum $M_{1}+M_{2}$ of two multifunctions $M_{1}$ and $M_{2}$ from $E$ into $F$ is defined by

$$
\left(M_{1}+M_{2}\right)(x)=M_{1}(x)+M_{2}(x) \text { for each } x \in E .
$$

3.6 Proposition. Let $M_{i}, i=1,2$, be two multifunctions from $E$ into $F$ which are additively separate at $\left(\bar{x} ; \bar{y}_{1}, \bar{y}_{2}\right)$. Then for each $\bar{v} \in E$,

$$
Q\left(M_{1} ; \bar{x}, \bar{y}_{1}\right)(\bar{v})+T\left(M_{2} ; \bar{x}, \bar{y}_{2}\right)(\bar{v}) \subset T\left(M_{1}+M_{2} ; \bar{x}, \bar{y}_{1}+\bar{y}_{2}\right)(\bar{v}) .
$$

Proof. Let $\bar{w}_{1}$ be a point in $Q\left(M_{1} ; \bar{x}, \bar{y}_{1}\right)(\bar{v})$ and let $\bar{w}_{2}$ be a point in $T\left(M_{2} ; \bar{x}, \bar{y}\right)(\bar{v})$. Consider a neighbourhood $V$ of $\bar{v}$ in $E$ and $W$ of $\bar{w}_{1}+\bar{w}_{2}$ in $F$. Choose neighbourhoods $W_{i}, i=1,2$, of $\bar{w}_{i}$ in $F$ such that $W_{1}+W_{2} \subset W$. Then, according to Definition 2.1 there exist a neighbourhood $X_{1}$ of $\bar{x}$ in $E$, a neighbourhood $Y_{1}$ of $\bar{y}_{1}$ in $F$, a real number $\varepsilon_{1}>0$ and a neighbourhood $V_{1}$ of $\bar{v}$ in $E$ with $V_{1} \subset V$ such that

$$
\left[\left(x, y_{1}\right)+t\left(\{v\} \times W_{1}\right)\right] \cap \operatorname{Gr} M_{1} \neq \varnothing
$$

for all $\left.\left(x, y_{1}\right) \in\left(X_{1} \times Y_{1}\right) \cap \operatorname{Gr} M_{1}, t \in\right] 0, \varepsilon_{1}\left[\right.$ and $v \in V_{1}$. On the other hand, it follows from Definition 1.2 that there exist a neighbourhood $X_{2}$ of $\bar{x}$ in $E$, a neighbourhood $Y_{2}$ of $\bar{y}_{2}$ in $F$, a positive number $\varepsilon<\varepsilon_{1}$ such that

$$
\left[\left(x, y_{2}\right)+t\left(V_{1} \times W_{2}\right)\right] \cap \operatorname{Gr} M_{2} \neq \varnothing
$$


for all $\left(x, y_{2}\right) \in\left(X_{2} \times Y_{2}\right) \cap \operatorname{Gr} M_{2}$ and $\left.t \in\right] 0, \varepsilon[$. Moreover, by Definition 3.1 there exist a neighbourhood $Y$ of $\bar{y}_{1}+\bar{y}_{2}$ in $F$ and a neighbourhood $X$ of $\bar{x}$ in $E$ with $X \subset X_{1} \cap X_{2}$ such that

$$
\left(M_{1}(x)+M_{2}(x)\right) \cap Y \subset Y_{1} \cap M_{1}(x)+Y_{2} \cap M_{2}(x)
$$

for all $x \in X$. Consider any point $(x, y) \in(X \times Y) \cap \operatorname{Gr}\left(M_{1}+M_{2}\right)$ and any number $t \in] 0, \varepsilon\left[\right.$. There exist by (19) two points $y_{i} \in Y_{i} \cap M_{i}(x), i=1,2$, satisfying $y=y_{1}+y_{2}$ and hence, according to (18), there exist $v \in V_{1} \subset V$ and $w_{2} \in W_{2}$ such that $y_{2}+t w_{2} \in M_{2}(x+t v)$. But (17) implies that there exists $w_{1} \in W_{1}$ such that $y_{1}+t w_{1} \in M_{1}(x+t v)$ and hence $y+t\left(w_{1}+w_{2}\right) \in\left(M_{1}+M_{2}\right)(x+t v)$. Therefore

$$
[(x, y)+t(V \times W)] \cap \operatorname{Gr}\left(M_{1}+M_{2}\right) \neq \varnothing,
$$

and the proof is complete.

The proof of the following proposition is similar to that of Proposition 3.6 and hence it will be omitted.

3.7 Proposition. Let $M_{i}, i=1,2$, be two multifunctions from $E$ into $F$ which are additively separate at $\left(\bar{x} ; \bar{y}_{1}, \bar{y}_{2}\right)$. Then for each $\bar{v} \in E$,

$$
Q\left(M_{1} ; \bar{x}, \bar{y}_{1}\right)(\bar{v})+Q\left(M_{2} ; \bar{x}, \bar{y}_{2}\right)(\bar{v}) \subset Q\left(M_{1}+M_{2} ; \bar{x}, \bar{y}_{1}+\bar{y}_{2}\right)(\bar{v}) .
$$

4. Composition of two multifunctions. Let $N$ be a multifunction from $E$ into $F$ and let $M$ be another multifunction from $F$ into a topological vector space $G$. We shall consider the multifunction $M^{-1}$ from $G$ into $F$ defined by

$$
M^{-1}(z)=\{y \in F: z \in M(y)\} \quad \text { for each } z \in G,
$$

and the multifunction $M \circ N$ from $E$ into $G$ defined by

$$
M \circ N(x)=\left\{z \in G: \exists y \in N(x) \cap M^{-1}(z)\right\}
$$

for each $x \in E$.

Let us recall the following definition.

4.1 Definition. The multifunction $M$ is said to be lower semicontinuous at $(\bar{x}, \bar{y}) \in \mathrm{Gr} M$ relative to a subset $D$ containing $\bar{x}$ if for each neighbourhood $Y$ of $\bar{y}$ in $F$ there exists a neighbourhood $X$ of $\bar{x}$ in $E$ such that $Y \cap M(x) \neq \varnothing$ for all $x \in D \cap X$.

REMARK. The reader will note that $M$ is lower semicontinuous at $\bar{x} \in E$ if and only if for each $\bar{y} \in M(\bar{x})$ the multifunction $M$ is lower semicontinuous at $(\bar{x}, \bar{y})$ relative to the whole space $E$.

4.2 Proposition. Let $M$ and $N$ be two multifunctions defined as above. Let $\bar{y} \in N(\bar{x})$ and let $\bar{z} \in M(\bar{y})$. If the multifunction from $E \times G$ into $F$ defined by $(x, z) \mapsto N(x) \cap M^{-1}(z)$ is lower semicontinuous at $((\bar{x}, \bar{z}), \bar{y})$ relative to $\operatorname{Gr} M \circ N$, then

$$
[Q(M ; \bar{y}, \bar{z}) \circ Q(N ; \bar{x}, \bar{y})](\bar{v}) \subset Q(M \circ N ; \bar{x}, \bar{z})(\bar{v})
$$

for all $\bar{v} \in E$.

Proof. Let $\bar{v}$ be a point in $E$, let $\bar{w}$ be a point in the first set of the preceding inclusion and let $W$ be a neighbourhood of $\bar{w}$ in $G$. There exists a point $\bar{u} \in F$ such 
that $\bar{u} \in Q(N ; \bar{x}, \bar{y})(\bar{v})$ and $\bar{w} \in Q(M ; \bar{y}, \bar{z})(\bar{u})$. By Definition 2.1 there exist a neighbourhood $Y_{1}$ of $\bar{y}$ in $F$, a neighbourhood $Z_{1}$ of $\bar{z}$ in $G$, a neighbourhood $U$ of $\bar{u}$ in $F$ and a real number $\varepsilon_{1}>0$ such that

$$
[(y, z)+t(\{u\} \times W)] \cap \operatorname{Gr} M \neq \varnothing
$$

for all $(y, z) \in\left(Y_{1} \times Z_{1}\right) \cap \operatorname{Gr} M, u \in U$ and $\left.t \in\right] 0, \varepsilon_{1}[$. By Definition 2.1 again there exist an open neighbourhood $Y$ of $\bar{y}$ in $F$ with $Y \subset Y_{1}$, a neighbourhood $X_{1}$ of $\bar{x}$ in $E$, a neighbourhood $V$ of $\bar{v}$ in $E$ and a positive number $\varepsilon<\varepsilon_{1}$ such that

$$
[(x, y)+t(\{v\} \times U)] \cap \operatorname{Gr} N \neq \varnothing
$$

for all $(x, y) \in\left(X_{1} \times Y\right) \cap \mathrm{Gr} N, v \in V$ and $\left.t \in\right] 0, \varepsilon[$. By our assumption about the semicontinuity at $((\bar{x}, \bar{z}), \bar{y})$ there exist a neighbourhood $X$ of $\bar{x}$ in $E$ with $X \subset X_{1}$, a neighbourhood $Z$ of $\bar{z}$ in $G$ with $Z \subset Z_{1}$ such that

$$
Y \cap N(x) \cap M^{-1}(z) \neq \varnothing
$$

for all $(x, z) \in(X \times Z) \cap \operatorname{Gr} M \circ N$. Consider a point $\left(x_{1}, z_{1}\right) \in(X \times Z) \cap$ Gr $M \circ N$, a point $v_{1} \in V$ and a number $\left.t_{1} \in\right] 0, \varepsilon$. Choose by (22) a point $y_{1} \in Y \cap N\left(x_{1}\right) \cap M^{-1}\left(z_{1}\right)$. There exists by (21) a point $u_{1} \in U$ such that

$$
y_{1}+t_{1} u_{1} \in N\left(x_{1}+t_{1} v_{1}\right) \text {. }
$$

But by (20) there exists a point $w_{1} \in W$ such that $z_{1}+t_{1} w_{1} \in M\left(y_{1}+t_{1} u_{1}\right)$ and hence, thanks to (23),

$$
z_{1}+t_{1} w_{1} \in M \circ N\left(x_{1}+t_{1} v_{1}\right)
$$

Therefore

$$
\left[\left(x_{1}, z_{1}\right)+t_{1}\left(\left\{v_{1}\right\} \times W\right)\right] \cap \operatorname{Gr} M \circ N \neq \varnothing
$$

and hence $\bar{w} \in Q(M \circ N ; \bar{x}, \bar{z})(\bar{v})$.

4.3 COROLLARY. Let $f$ be a mapping from $E$ into $F$ which is strictly differentiable at $\bar{x} \in E$ with derivative $\nabla f(\bar{x}):=A$, let $M$ be a multifunction from $F$ into $G$ and let $\bar{z}$ be a point in $M(f(\bar{x}))$. Then

$$
Q(M ; f(\bar{x}), \bar{z})(A \bar{v}) \subset Q(M \circ f ; \bar{x}, \bar{z})(\bar{v})
$$

for all $\bar{v} \in E$.

Proof. Since $f$ is continuous at $\bar{x}$, it is not difficult to see that the multifunction $(x, z) \mapsto\{f(x)\} \cap M^{-1}(z)$ is lower semicontinuous at $((\bar{x}, \bar{z}), f(\bar{x}))$ relative to $\mathrm{Gr} M \circ f$, and hence the corollary is a direct consequence of Proposition 4.2 and Corollaries 1.7 and 2.11 .

Analogously to Proposition 4.2 one can show the following result, whose proof is left to the reader.

4.4 Proposition. Let $M$ and $N$ be two multifunctions verifying the assumptions of Proposition 4.2. Then

$$
[Q(M ; \bar{y}, \bar{z}) \circ T(N ; \bar{x}, \bar{y})](\bar{v}) \subset T(M \circ N ; \bar{x}, \bar{z})(\bar{v})
$$

for all $\bar{v} \in E$. 
As a direct consequence of Propositions 4.4 and 2.9, we have the following result.

4.5 COROLlaRY. If the multifunctions $M$ and $N$ verify the assumptions of Proposition 4.2 and if $M$ is lipschitzian at $\bar{y}$ in any direction, then for all $\bar{v} \in E$,

$$
[T(M ; \bar{y}, \bar{z}) \circ T(N ; \bar{x}, \bar{y})](\bar{v}) \subset T(M \circ N ; \bar{x}, \bar{z})(\bar{v}) .
$$

\section{REFERENCES}

1. J. P. Aubin, Contingent derivatives of set-valued maps and existence of solutions to nonlinear inclusions and differential inclusions, Math. Res. Center Technical Summary Report No. 2044, Univ. of Wisconsin, Madison, 1980.

2. C. Berge, Espaces topologiques, fonctions multivoques, Dunod, Paris, 1959.

3. J. M. Borwein, A Lagrange multipler theorem and a sandwich theorem for convex relations, Math. Scand. 48 (1981), 189-204.

4. __ Multivalued convexity; a unified approach to equality and inequality constraints, Math. Programming 13 (1977), 163-180.

5. F. H. Clarke, Generalized gradients and applications, Trans. Amer. Math. Soc. 205 (1975), 247-262.

6. __ A new approach to Lagrange multipliers, Math. Oper. Res. 1 (1976), 165-174.

7. S. Dolecki, Tangency and differentiation: some applications of convergence theory, Ann. Mat. Pura Appl. (to appear).

8. P. C. Duong and H. Tuy, Stability, surjectivity, and local invertibility of non differentiable mappings, Acta Math. Vietnam. 3 (1978), 89-105.

9. S. Gautier, Différentiabilité des multiapplications, Publ. Math. de Pau 5 (1978), 1-17.

10. J. B. Hiriart-Urruty, Tangent cones, generalized gradients and mathematical programming in Banach spaces, Math. Oper. Res. 4 (1979), 79-97.

11. J. B. Hiriart-Urruty and L. Thibault, Existence et caractérisation de différentielles généralisées d'applications localement lipschitziennes d'un Banach séparable dans un Banach réflexif séparable, $\mathrm{C}$. $\mathrm{R}$. Acad. Sci. Paris Sér. I. Math. 290 (1980), 1091-1094.

12. A. D. Ioffe, Nonsmooth analysis: differential calculus of nondifferentiable mappings, Trans. Amer. Math. Soc. 266 (1981), 1-56.

13. J. L. Kelley, General topology, Springer-Verlag, New York, 1975.

14. S. Mirica, The contingent and the paratingent as generalized derivatives for vector-valued and set-valued mapping (to appear).

15. J. P. Penot, Differentiability of relations and differential stability of perturbed optimization problems (to appear).

16. On regularity conditions in mathematical programming, Math. Programming Stud. 19 (1982), 167-199.

17. J. P. Penot and M. Thera, Polarité des applications convexes à valeurs vectorielles, C. R. Acad. Sci. Paris Sér. I Math. 288 (1979), 419-422.

18. A. L. Peressini, Ordered topological vector spaces, Harper \& Row, New York, 1967.

19. S. Robinson, Normed convex processes, Trans. Amer. Math. Soc. 174 (1972), 127-140.

20. Regularity and stability for convex multivalued functions, Math. Oper. Res. 1 (1976), 130-143.

21. R. T. Rockafellar, Convex analysis, Princeton Univ. Press, Princeton, N.J., 1970.

22. __ Clarke's tangent cones and the boundaries of closed sets in $\mathbf{R}^{n}$, Nonlinear Anal. 3 (1979), $145-154$

23. Generalized directional derivatives and subgradients of nonconvex functions, Canad. J. Math. 32 (1980), 257-280.

24. __ Directionally lipschitzian functions and subdifferential calculus, Proc. London Math. Soc. 39 (1979), 331-355.

25. L. Thibault, Problème de Bolza dans un espace de Banach séparable, C. R. Acad. Sci. Paris Sér. I Math. 282 (1976), 1303-1306.

26. (1980), 157-192. , Subdifferentials of compactly lipschitzian vector-valued functions, Ann. Mat. Pura Appl. 125

27. Mathematical programming and optimal control problems defined by compactly lipschitzian mappings, Sem. Analyse Convex, Exp. no. 10, Montpellier, 1978. 
28. __ On generalized differentials and subdifferentials of Lipschitz vector-valued functions, Nonlinear Analysis Th. Math. Appl. 6 (1982), 1037-1053.

29. $319-354$.

30. E__ Epidifférentiels de fonctions vectorielles, C. R. Acad. Sci. Paris Sér. I Math. 290 (1980), 87-90.

31. __ Lagrange multipliers for nonconvex multifunctions (to appear).

32. C. Ursescu, Multifunctions with closed convex graph, Czechoslovak Math. J. 25 (1975), 438-441.

Departement de Mathematiques, Universite de Pau, 64000-Pau, France 